\title{
EchoGéo
}

$7 \mid 2008$

Les nouveaux enjeux régionaux dans l'océan Indien occidental

\section{La participation des acteurs locaux à l'élaboration et l'évaluation des politiques publiques de développement durable en Nouvelle-Calédonie}

Séverine Bouard, Jean-Michel Sourisseau et Gilles Pestana

\section{(Q) OpenEdition}

Journals

Édition électronique

URL : https://journals.openedition.org/echogeo/9693

DOI : 10.4000/echogeo.9693

ISSN : 1963-1197

Éditeur

Pôle de recherche pour l'organisation et la diffusion de l'information géographique (CNRS UMR 8586)

Référence électronique

Séverine Bouard, Jean-Michel Sourisseau et Gilles Pestana, «La participation des acteurs locaux à

l'élaboration et l'évaluation des politiques publiques de développement durable en Nouvelle-

Calédonie », EchoGéo [En ligne], 7 | 2008, mis en ligne le 25 novembre 2008, consulté le 31 juillet 2021.

URL : http://journals.openedition.org/echogeo/9693; DOI : https://doi.org/10.4000/echogeo.9693

Ce document a été généré automatiquement le 31 juillet 2021.

EchoGéo est mis à disposition selon les termes de la licence Creative Commons Attribution - Pas d'Utilisation Commerciale - Pas de Modification 4.0 International (CC BY-NC-ND) 


\title{
La participation des acteurs locaux à l'élaboration et l'évaluation des politiques publiques de développement durable en Nouvelle-Calédonie
}

\author{
Séverine Bouard, Jean-Michel Sourisseau et Gilles Pestana
}

\section{Introduction}

1 L'objet de cet article est d'explorer les conditions et l'opérationnalité d'une réflexion participative sur le développement durable et ses implications pour élaborer et évaluer des politiques publiques de développement. Le développement durable y est perçu comme un concept qui doit être contextualisé plutôt que comme un référentiel global. Nous faisons l'hypothèse que pour qu'il puisse alimenter efficacement la réflexion sur les politiques publiques, il doit être (re)défini en tenant compte des caractéristiques et des spécificités du territoire concerné. Pour cela, nous pensons qu'une approche participative est nécessaire, qui doit s'attacher au renforcement des capacités des acteurs locaux et respecter quelques principes de base.

2 Après une présentation succincte des controverses dont fait l'objet le concept de développement durable à l'échelle mondiale, nous tenterons de préciser les enjeux de durabilité dans le contexte de la Nouvelle-Calédonie. Pour illustrer ces enjeux, nous nous intéressons plus précisément à la gestion sociale des impacts d'un important projet industriel d'extraction et de traitement du nickel dans la province Nord de Nouvelle-Calédonie. Ce projet d'envergure à l'échelle d'une population réduite est aussi la pierre angulaire de la politique publique de développement de l'exécutif provincial. La gestion de ses impacts environnements, économiques et sociaux est un défi complexe de part la transversalité des enjeux. 
3 L'article tente alors de décrire les principes à respecter pour la mise en place d'un suivi évaluation concerté entre les différents acteurs, susceptible de faciliter la prise en charge de cette transversalité et la gestion sociale des impacts du projet. Nous partons pour cela des acquis d'un programme de recherche portant sur l'élaboration d'une méthode de suivi évaluation participatif des programmes de développement local en province Nord.

4 Enfin, nous insisterons sur les contraintes fortes à l'efficacité et à la pérennité de dispositif réellement participatif, mais aussi à l'application des principes du développement durable, notamment dans le contexte du Pacifique insulaire.

\section{Développement durable : un concept a priori unificateur mais controversé}

5 Le concept de développement durable est une notion ambiguë et paradoxale; objet d'un large consensus apparent depuis la définition proposée dans le rapport Brundlant en 1987 (CMED 1987), son intégration et sa traduction dans les politiques internationales, nationales et régionales sont diffuses, très inégales selon les situations, mais finalement décevantes (Aubertin et Vivien 2006).

6 L'acceptation politique du développement durable par les institutions internationales et, par suite, par la plupart des gouvernements nationaux, s'accommode finalement d'adaptations des politiques publiques traditionnelles, sans en remettre en cause les fondements. Le changement de paradigme attendu de l'émergence des concepts d'écodéveloppement puis de durabilité n'a pas eu lieu, parce que ce changement aurait été contraire aux intérêts et référentiels dominants (Figuière 2006), mais aussi, de façon plus pratique, parce qu'il supposait une difficile car radicale réorganisation des administrations et appareils d'Etat. Les faits montrent qu'un «verdissement » des politiques économiques et sociales en vigueur a été préféré à une refonte complètes de principes publics d'intervention (Aubertin et Vivien 2006).

7 La seconde raison, articulée à la première, est que le développement durable n'est pas un concept stabilisé et qu'il est l'objet de nombreuses controverses.

8 D'un point de vue conceptuel, le développement durable agite nombre de débats. Sa formulation par les agences internationales et la littérature institutionnelle qui l'accompagne en font un nouveau principe unificateur (Tubiana 2000), alors même que son caractère novateur fait débat. Pour les tenants du discours néolibéral l'introduction du développement durable offre en effet un nouveau champ de validation de théories anciennes et dominantes. Ils montrent, en justifiant une position dite de «durabilité faible » la capacité des régulations de marché à gérer les actifs environnementaux, les contraintes environnementales et les perspectives de durabilité de la seule croissance économique (Solow1999, Grossman et Krugger 1995). Le courant de l'économie écologique invite quant à lui à un recours aux sciences de la nature pour appréhender les spécificités des actifs environnementaux (Ehrlich 2008). La place relative effectivement accordée par les politiques aux dimensions économiques, environnementales et sociales du développement est également questionnée. La prédominance des questions environnementales semble préjudiciable à la résolution des problématiques sociales ; ainsi les résultats finalement rapides - quoiqu'inachevés - 
autour du protocole de Kyoto contrastent avec le creusement des inégalités dans les espaces nationaux comme à l'échelle internationale (Figuière 2006).

Ces débats théoriques rejoignent des débats méthodologiques fournis sur les objectifs concrets de politiques relevant de la définition substantive et intégrée (celle du rapport Brundlant) du développement durable. Par exemple, les travaux sur les indicateurs de durabilité, pourtant très nombreux, sont loin d'être aboutis (Rey-Valette et Roussel 2006). Les formes renouvelées de gouvernance que le développement durable était supposé impliquer tardent à se mettre en place. Derrière l'encouragement apparent à davantage de participation citoyenne, on assiste plutôt à une normalisation des messages à l'échelle internationale.

Dans le champ idéologique, la traduction politique du concept de développement durable est fortement critiqué (Harribey 2002, Godard 2004, Latouche 2004). L'encouragement des logiques privilégiant la croissance est d'une part dénoncé, l'intégration des préoccupations environnementales se révélant finalement peu contraignante. Plus encore, sous couvert d'une prise en compte de la finitude du monde, les traductions onusiennes et des ONG internationales de la durabilité ne feraient qu'entretenir et préserver la domination de l'occident, le plus grand pollueur devenant le donneur de leçon en matière d'environnement. L'ingérence écologique est en particulier dénoncée comme un moyen de réduire les perspectives de développement des pays émergents, et plus encore des pays en développement (Rossi 2000). La réflexion sur le développement durable se déplace alors vers la question plus globale et finalement déjà ancienne du « développement » en lui-même (Rist 1996, Berr et Harribey 2005).

\section{La problématique du développement durable dans le débat calédonien}

11 En Nouvelle-Calédonie, archipel de $19100 \mathrm{~km}^{2}$ pour $240000 \mathrm{hab}$., toutes les conditions sont réunies pour que le développement durable dans sa version substantive onusienne ait du succès. Le territoire connaît un développement économique rapide porté par l'industrialisation de son activité minière ${ }^{1}$, son environnement naturel réputé fragile est plébiscité pour la richesse de sa biodiversité marine et terrestre et son fort endémisme ${ }^{2}$, elle est aussi engagée dans un processus original d'autonomisation politique depuis la fin des années 1980 .

\subsection{Un concept émergeant via des acteurs externes}

12 Le développement durable est aujourd'hui très présent chez les acteurs publics et privés calédoniens. Force est de constater cependant que les premiers qui s'en sont saisis sont des acteurs externes. Les firmes multinationales canadiennes (Inco et surtout Falconbridge, promoteurs initiaux des nouveaux projets industriels, aujourd'hui Vale et Xstrata, leurs repreneurs), sont rodées depuis plusieurs années - et de plus en plus surveillés - aux principes de responsabilité sociale et environnementale dans leur pratique industrielle; elles ont apporté des notions de durabilité dans les activités extractives. Les grandes ONGs internationales environnementales (UICN, WWF, CI) interviennent de plus en plus du fait de la médiatisation croissante de l'archipel; elles ont aussi apporté une rhétorique "classique» du développement 
durable dans les approches environnementales. Ces acteurs externes sont implantés dans la durée en Nouvelle-Calédonie et bénéficient de partenariat devant garantir leur ancrage local ; ils conservent néanmoins leurs logiques propres et reproduisent en des normes du développement durable qu'ils mobilisent partout ailleurs.

\subsection{Développement durable, destin commun et rééquilibrage} durable dans la sphère des politiques publiques mérite une réflexion critique. En premier lieu, le risque d'une focalisation de l'attention des politiques sur les problématiques environnementales alors que d'importantes questions sociales restent très prégnantes est indéniable. Ce risque est aggravé par les conditions d'émergence du concept et pourrait s'accompagner d'une normalisation des messages sur la durabilité, contribuant à une décontextualisation du concept.

Cette normalisation doit aussi être discutée dans le cadre de l'affirmation parallèle des notions de destin commun et de rééquilibrage. Ces dernières, issues du règlement politique des Evénements des années $1980^{3}$, dominent la question du développement et de la construction des politiques publiques depuis près de 20 ans (Christnacht, 2004.). Elles renvoient à des objectifs forts de partage communautaire du pouvoir, à une revalorisation de la culture kanak, au rattrapage volontariste des retards de développement des zones et populations hors Nouméa. Le développement durable peut apparaître comme porteur des mêmes principes et son ambition intégratrice offre a priori une opportunité de (re)donner un sens et une justification conceptuelle aux concepts moteurs des politiques publiques et du lien avec la France depuis 1989. Cependant, si à la différence du développement durable, destin commun et rééquilibrage résultent d'un compromis local et sont profondément ancrés dans les réalités propres de l'archipel, ils furent longs à se dessiner et leur efficacité est aujourd'hui remise en cause par une partie de l'opinion publique. Dans ce contexte, on ne peut exclure leur affaiblissement face à l'hégémonie d'un référentiel externe, plus lisible dans les sphères internationales mais pouvant à terme légitimer un retour à des clivages anciens porteurs d'instabilités.

\subsection{Le développement durable dans le discours}

Lorsque l'on interroge un panel de décideurs, de responsables d'associations et d'administrations sur leur représentation du développement durable, on note des divergences particulièrement marquées. Si tous expriment le besoin d'articulation entre les dimensions environnementale, économique et sociale (y compris culturelle), ils hiérarchisent différemment les pôles et mettent en avant des interactions de nature parfois opposée. Très schématiquement, les discours reproduisent aujourd'hui, parfois de façon très tranchée, les clivages politiques, économiques et sociaux qui prévalaient lors de la sortie de crise des années 1980 ; les divergences se superposent aux regards positifs ou plus mitigés portées sur la notion de destin commun. L'introduction du référentiel du développement durable dans les discours peut alors contribuer à s'éloigner du référentiel spécifique issu du compromis des accords politiques locaux. 


\section{Les politiques publiques néo-calédoniennes}

\subsection{Les politiques publiques de développement de la Province Nord articulées à un grand projet industriel}

En Province Nord, gérée par des élus indépendantistes, une des composantes centrales de la politique de rééquilibrage est la construction d'une usine de transformation du nickel. Pour la réalisation de ce projet industriel, l'exécutif provincial a fait des choix qui doivent lui permettre de garder la maîtrise de l'opération et d'en contrôler les impacts. Pour conduire un projet d'une telle ampleur, il a dû conclure un accord de partenariat avec une multinationale. Il a réussi à négocier de conserver $51 \%$ du consortium chargé de la mise en œuvre du projet; ceci lui assure, par suite, $51 \%$ des dividendes. Les choix politiques provinciaux en matière de développement durable s'expriment alors à plusieurs niveaux autour de ce projet. Une attention sans précédent est d'abord portée à l'impact environnemental du projet industriel, attention qui contraste avec les habitudes calédoniennes. Refusant la logique "d'enclave », le projet est aussi conçu comme un moyen au service du développement économique et social provincial. Au-delà des 2700 emplois directs et indirects prévus durant plus de 30 ans, des mesures sont prises pour favoriser une diffusion de la croissance économique induite, pour diversifier l'économie, pour préparer l'après-nickel, pour améliorer les services publics, etc. Du fait de son envergure, le projet va de toute façon accélérer les processus de changement auxquels sont déjà soumis les 45000 habitants de la Province Nord.

17 Ainsi, parallèlement aux questions cruciales de garantie locale de l'emploi, de maximisation des retombées économiques et de minimisation des impacts environnementaux, la maîtrise des effets du projet industriel sur la population, et donc leur gestion par les différentes parties prenantes constituent des enjeux considérables. Pour éclairer les formes d'accompagnement qui seraient nécessaires, une étude sociologique, commanditée par la Province Nord, a été réalisée en 2005 et 2006. (Sourisseau et al., 2006)

\subsection{La gestion sociale des impacts du projet Koniambo}

18 Les principaux résultats de cette étude montrent que les populations locales comprennent la place centrale du projet minier dans la stratégie provinciale de développement, ce qui fait que le projet industriel bénéficie d'un soutien fort de la population. Cependant, malgré ce soutien, les populations locales ont de réelles inquiétudes et attentes...Les tensions vont apparaître inévitablement au fur et à mesure de l'avancement du projet c'est pourquoi il importe de les prévenir le plus possible en anticipant et facilitant la construction de compromis négociés autour des questions que se pose la maîtrise du projet.

19 Parmi les principaux défis qui devraient faire l'objet d'une attention particulière, on peut citer (Sourisseau et al., 2006) : l'accès aux emplois salariés et la gestion des questions environnementales. Pour ces deux premières questions, les acteurs locaux demandent une transparence accrue dans les procédures et la circulation de l'information. Ensuite, la population locale souhaite que soit maîtrisée et renforcée la cohésion sociale. L'accélération du changement économique aura des effets importants 
sur les formes de régulation sociale existant dans les tribus, où vit la majorité de la population kanak.

Ainsi, les enjeux cités par la population locale sur le projet Koniambo rejoignent les enjeux du référentiel des politiques publiques de la Province Nord qui cherchent à construire d'un développement adapté aux sociétés locales autour du projet Koniambo. On voit bien qu'au niveau global du territoire comme au niveau local du rayonnement de l'usine, se posent des problèmes importants de mise en débat des enjeux, des craintes et attentes des différentes catégories de la population, de partage des décisions et de l'évaluation du projet, pour accompagner au mieux le développement. Bref, on est bien au cœur de la recherche de principes de gouvernance adaptés au contexte spécifique de la Nouvelle-Calédonie et de son histoire politique (marquée par la domination). La recherche d'une gouvernance locale adaptée est encore plus stratégique dans ce contexte de multiplication des acteurs sur un si petit territoire où la culture de la participation et la gouvernance locale est peu développée.

21 Cette question de la gouvernance locale sur un projet comme le Koniambo renvoie plus largement aux débats sur le développement durable et sur sa contextualisation : audelà des divergences exprimées, la recherche de compromis négociés est un dénominateur commun des acteurs interrogés sur leur perception de la durabilité. Répondre aux défis signalés et à l'enjeu de «bonne gouvernance " passe, selon nous, par la mise en œuvre d'approches méthodologiques réellement participatives. La recherche finalisée a son rôle à jouer et peut contribuer à construire, tester, expérimenter certaines de ses approches. Plus concrètement, les résultats obtenus ces dernières années (d'Aquino, 2005, Bouard et al, 2006) permettent de proposer des éléments de cadrage méthodologique pour la mise en place de suivi-évaluation participatif des politiques publiques intégrant le projet minier.

\subsection{Les acquis d'une expérimentation de suivi participatif}

Entre 2003 et 2005, a été conçue et expérimentée dans le Nord Est de la Nouvelle Calédonie une démarche participative pour procurer aux acteurs locaux les moyens de suivre et d'évaluer un programme de développement local, l'Opération groupée d'aménagement du foncier de la Côte Est.

La méthode choisie ne correspondait pas à une évaluation externe classique, mais entre dans le cadre des outils de suivi participatif expérimentés par les institutions internationales d'appui aux projets de développement (Fetterman et al 1996, Harnmeijer, 1999, Davies et Dart 2005, Estrella et al 2000). L'entrée privilégiée a été l'analyse et le suivi de l'Ogaf Côte Est. L'enjeu était de faire remonter par les acteurs eux-mêmes, au moyen d'ateliers participatifs, leurs propres enjeux concernant le développement de la région, les apports de l'opération et les contraintes qu'elle rencontre. Outre l'analyse des effets du projet, il s'agissait aussi de réfléchir avec les institutions et les acteurs ciblés à la durabilité des effets induits du programme.

Pour notre part, procurer aux acteurs locaux les moyens et les capacités permettant d'effectuer un suivi de qualité passe par un accroissement des " capacités sociales » des acteurs à participer (d'Aquino et al. 1999, d'Aquino 2005, Bouard et al, 2006). Pour cela la démarche s'est déroulée en trois étapes : une phase de légitimation sociale, une phase de construction des capacités techniques à prendre en charge des 
questionnements complexes, une phase d'obtention des capacités stratégiques à influencer les modes de décision.

La phase initiale de "légitimation sociale» a pour objectif d'accompagner la construction de la légitimité des différents acteurs, reconnue par lui-même et par les autres, à s'impliquer dans la prise de décision. Pour tenter d'atteindre ces objectifs, des ateliers séparés ont été organisés avec chaque type d'acteur : agriculteurs, la société civile (essentiellement associations), les mairies. Lors de ces ateliers, il était demandé aux participants de se positionner sur les enjeux de développement de la Côte Est, de leur institution de rattachement, puis du programme de développement OGAF Côte Est). Ces derniers concernaient schématiquement : le développement de l'agriculture, l'installation des agriculteurs, l'autonomisation des agriculteurs, l'adaptation des politiques de développement par rapport à la culture kanak.

Pour la phase d'obtention des capacités techniques à prendre en charge collectivement un questionnement soulevé par les politiques publiques, il s'agit de développer les ressources permettant aux acteurs participant au suivi d'exprimer leur point de vue. Cette étape s'est traduite par la fourniture de l'information utile au suivi par ces acteurs de la pertinence et de l'efficacité du programme sur les enjeux qu'ils ont identifiés en première phase comme prioritaires et la mise au point de formes d'analyse et de restitution participatives de cette information, adaptées aux acteurs visés (voir infra). Pour le développement de l'agriculture, par exemple, des enquêtes socio économiques sur les stratégies des ménages ont été réalisées pour décrire les facteurs et discuter les contraintes du développement de l'agriculture en milieu kanak (Sourisseau et al. 2005).

Pour donner aux acteurs locaux les moyens d'effectuer une analyse fine des programmes de développement, il s'agissait de concevoir un outil participatif de suivi du programme assez précis pour être pertinent et assez lisible pour être utilisable auprès des différents types d'acteurs, en particulier en tribu. Le support devait donc essayer de s'appuyer sur les références culturelles, être lisible par tous mais gardant la complexité des faits, se corriger aisément, faciliter l'interactivité et enfin être évolutif ${ }^{4}$. (cf. figure 1) 


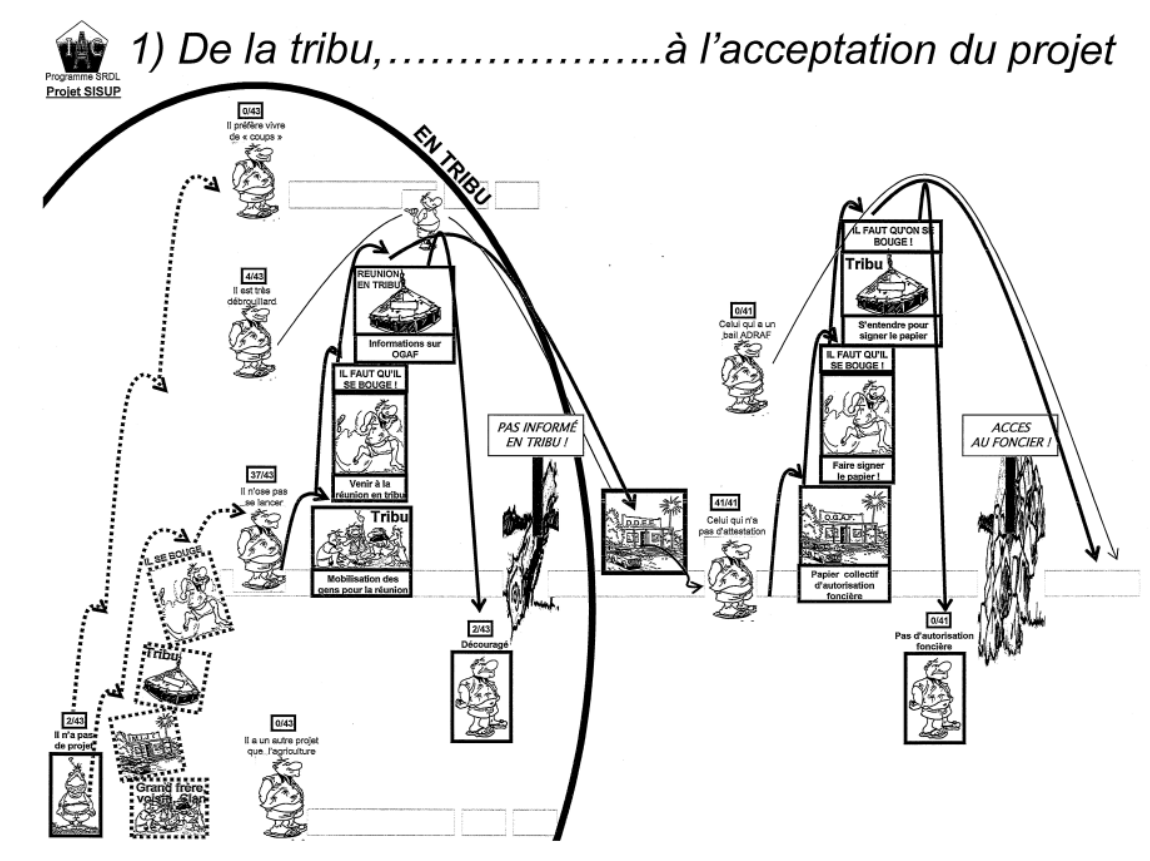

Source : Bouard et al., 2006 services d'appui techniques et les responsables politiques? C'est une contrainte qui concerne toutes les démarches participatives et qui fait l'objet de la dernière phase de la démarche présentée (d'Aquino, 2002). Il s'agissait de construire une méthode permettant de renforcer les interactions entre les différents niveaux décisionnels micro et macro. Pour cela, nous avons centré la troisième phase de la démarche sur l'émergence d'un réseau informel composé d'acteurs locaux affiliés à différentes institutions, du micro au macro, mais partageant les mêmes enjeux et la volonté de faire diffuser le constat issu du suivi. Un groupe de personnes ressources rencontrées 
au cours des ateliers se retrouve donc fréquemment dans le but de traduire progressivement les souhaits émis par les acteurs en propositions concrètes pour les politiques publiques de développement.

\section{Principes méthodologiques pour construire et évaluer de façon participative les politiques de développement durable de la Province Nord}

31 La littérature sur les politiques publiques de développement durable et cette expérimentation nous permettent donc de proposer une approche pour accompagner une réflexion participative sur le développement durable en Nouvelle-Calédonie. Pour notre part comme pour un certain nombre d'auteurs (Godard, 1994, Vivien, 2004, Revéret, 2004), il est tout d'abord important de mettre en place une démarche anthropocentrée et privilégiant une " entrée " sociale ; c'est-à-dire une démarche basée sur la définition des objectifs du développement durable selon le «bien-être » (Hatem, 1990) des habitants de la province Nord et tels que perçus par eux. Ensuite, une réflexion sur les politiques de développement durable nécessite de créer une articulation entre les différents niveaux territoriaux d'application des politiques publiques. Il s'agit de favoriser la rencontre des perceptions locales et globales du développement durable et ainsi tenter de limiter les problèmes liés à l'application du concept conçu comme un référentiel global. Ainsi, concrètement, ces orientations laissent à penser qu'une démarche de suivi-évaluation participatif des politiques publiques de développement autour du projet Koniambo pourrait être pertinente.

\subsection{La construction des " capacités sociales »}

Comme pour l'exemple présenté, il s'agirait de concevoir une démarche basée sur les trois étapes présentées ci-dessus, avec comme objectif stratégique de construire les "capacités sociales" des acteurs (art.cit). La première phase viserait à renforcer la légitimité des différents acteurs à participer au suivi par la construction d'un cadre commun d'analyse des politiques publiques intégrant le projet Koniambo. Concrètement, pour cela chaque groupe d'acteurs devrait formaliser ces enjeux vis-àvis du projet, dresser un état des lieux des impacts attendus et de la façon dont l'industriel et la Province Nord pensent les gérer. Les ateliers pourraient être organisés auprès des acteurs suivants : les opérateurs privés (agriculteurs, artisans, commerçants, etc.), la société civile (essentiellement les associations), les mairies, les institutions provinciales. Il s'agit de laisser le débat ouvert, d'accompagner la définitions des enjeux de développement durable selon «leur bien-être » tels qu'ils le perçoivent, qu'il soit basé sur des critères sociaux, environnementaux ou économiques.

La deuxième phase se concentrerait sur la définition des indicateurs à suivre, la collecte de l'information selon les enjeux définis par les acteurs lors de la phase précédente. En parallèle, les informations collectées seraient restituées au fur et à mesure de leur capitalisation avec un important travail de construction de supports de discussions adaptés à la culture locale et aux problématiques.

La troisième phase aurait pour objectif de poursuivre la mobilisation collective avec les coutumiers, les mairies, les tribus, la société civile. Il s'agit de construire un niveau 
intermédiaire permettant d'accroître les liens d'influence avec les lieux de décision et d'améliorer les conditions d'un transfert effectif des conclusions du suivi en matière de développement vers les principaux intéressés.

Cependant, si la première expérience de suivi-évaluation participatif a ouvert des perspectives en terme de démarche; elle a par ailleurs montré que ces approches sont extrêmement dépendantes des contextes culturel et politique de la Nouvelle-Calédonie. Or, la démarche proposée n'a pas pour objectif de bouleverser l'organisation sociale, ni les résultats, à imposer des modèles exogènes; c'est pourquoi il faudra veiller à ce que la démarche participative et les analyses qui en découleront soient "culturellement acceptables" (Figuière, 2006). C'est l'objet de cette dernière partie qui présente les limites possibles à la mise en place d'une gouvernance locale pour le suivi-évaluation des politiques publiques. Ces éléments devront faire l'objet d'une attention particulière pour adapter au mieux les principes méthodologiques proposés.

\subsection{La nécessaire prise en compte du contexte socio-politique}

\subsubsection{Les caractéristiques de la société kanak face aux pratiques de la participation publique occidentale}

Dans la société kanak, la parole et le discours tiennent une place particulière. Cette place est tout d'abord visible dans le cérémoniel qui lui est accordé au moment des " coutumes » où le discours est le moment privilégié pour exposer les généalogies des clans (leur origine, leurs déplacements, les conflits, les alliances, etc.). Plus quotidiennement, l'accès à la parole dans la société kanak est soumis à de nombreuses règles (Bensa et al., 1985). Le processus collectif d'information, de négociation et de prise de décision est considéré très différemment des approches participatives publiques occidentales. En effet, lorsqu'un kanak s'exprime publiquement, sa propre parole engage et représente tous ses ancêtres mais elle concerne également tous les ancêtres de celui à qui il s'adresse ou dont il parle. Il est alors délicat d'exprimer publiquement un désaccord, de même que de contester le discours de quelqu'un. Le débat et la discussion orale publique à l'occidentale, qui impliquent une remise en cause au moins partielle du discours de l'interlocuteur, représentent donc dans ce contexte un mode d'expression qui peut s'interpréter comme un échange d'offenses sacrilèges envers les interlocuteurs et leurs ancêtres. La prise de parole lors des réunions coutumières est donc soigneusement mesurée, réservée à ceux ayant un statut élevé, et le débat y est délicat. Ainsi, lorsqu'il existe un besoin de débat ou de négociations, une bonne partie de la discussion se passe via des échanges individuels complémentaires aux prises de parole publiques et formelles.

Cependant, les Kanak maîtrisent aussi les arènes de discussion publiques introduites par la colonisation : conseil municipal, réunion associative, réunion politique,... Dans ces espaces collectifs, les codes culturels de l'expression en public y sont moins marqués que dans les espaces culturels et le débat y est plus répandu. Il y a donc, comme dans toute société, des espaces sociaux périphériques, moins codifiés qui aident à la prise en compte des changements par la société. L'idée est alors de laisser les acteurs locaux utiliser ces espaces de parole de la façon la plus pertinente, à leur rythme et selon les modes de communication qui y sont reconnus. Il s'agira de laisser les acteurs locaux maitres de leurs enjeux et de leurs modes de communication tout en 
les accompagnant dans la construction progressive de la problématique à prendre en charge.

\subsubsection{Un contexte politique particulier ne facilitant pas l'implication des populations et de leurs porte-paroles}

Le suivi évaluation participatif est souvent synonyme d'ouverture, d'implications de nouveaux acteurs en particuliers locaux qui amènent à un partage des compétences et du pouvoir souvent délicat. Dans ce sens, la première expérience de suivi évaluation participatif a été marquée par de nombreux allers-retours entre le niveau micro (bénéficiaires, autorités locales impliquées dans le programme, équipe en charge du programme de développement, partenaire du programme) et le niveau macro (directions techniques et élus décideurs de l'orientation des politiques publiques de développement).Les supports de discussion permettaient de juger le programme de développement en lui-même mais aussi de juger des tendances prises par la mise en application des politiques publiques de développement rural en Nouvelle-Calédonie. Ces premières expériences ont montré la difficulté à mettre en œuvre une démarche qui favorise la prise en compte des perceptions locales des problématiques ainsi que des décisions locales par les niveaux hiérarchiques supérieurs.

39 L'implication dans les politiques de développement des populations et de leurs portes paroles de terrain, y compris communaux, est en effet encore peu répandue en Nouvelle-Calédonie. Avec une histoire politique opposant indépendantistes et loyalistes, la décision est traditionnellement très hiérarchisée, tous les pouvoirs ont tendance à être tacitement attribués aux niveaux supérieurs de chacune des deux parties. De plus, dans ce pays en construction, les autorités provinciales sont encore un peu réticentes à partager le pilotage du développement, qu'elles construisent progressivement, avec les communes et les populations. Les populations, les coutumiers et les communes se sentent alors encore peu légitimes à intervenir dans le choix des politiques de développement.

Une attention particulière sera portée à la concertation entre les différents niveaux. Il s'agira de toucher différents niveaux décisionnels et mais également prévoir des interactions entre différentes échelles de décisions du micro (acteurs locaux) au macro (politiques nationales, internationales, etc.), de favoriser les négociations, de travailler sur des supports adaptés pour ainsi faire fonctionner de concert et dans la durée des acteurs et institutions ayant des objectifs et des modes de gouvernance propres.

\section{Conclusion}

41 La mise en œuvre du projet Koniambo, projet industriel d'envergure de transformation $\mathrm{du}$ nickel, offre une occasion particulièrement fertile de discuter plus largement des enjeux de durabilité en Nouvelle-Calédonie. On l'a vu, concilier les principes du développement durable tout en renforçant le référentiel du destin commun et du rééquilibrage est un défi méthodologique et conceptuel majeur. Les travaux de recherche finalisée de l'Institut Agronomique néo-Calédonien offrent des perspectives pour suivre au plus près et accompagner la construction de nouveaux modes de gouvernance, la mise en agenda politique du développement durable et l'efficacité des mesures qui seront prises. 

locales à des contingences externes. Dans le contexte particulier du développement industriel minier en province Nord, l'accord global sur le projet de l'usine du Nord, le niveau de vie élevé de la population, le volontarisme de la puissance publique, les précautions prises avec la participation majoritaire de la Province et l'arbitrage de l'Etat français laissaient à penser que la maîtrise locale du projet était acquise. Or, les rachats dans le secteur du nickel sous la pression des actionnaires et les tergiversations des multinationales engagées dans un calcul mondial ont déjà occasionné près de 10 ans de retard dans la construction de l'usine du Nord. Ces retards nous rappellent que la construction de politiques publiques de développement durable est influencée par l'importation de référentiels portée par des macro-acteurs, mais que ces mêmes acteurs peuvent aussi freiner la mise en place de politiques qu'ils ont eux-mêmes contribué à élaborer. La dépendance au contexte mondial est donc réelle et fragilisante; elle peut gêner la participation des populations en ajoutant de l'incertitude et en entretenant le doute sur les intentions réelles du partenaire industriel.

Ainsi la contextualisation souhaitée des fondements constituant du développement durable s'avère difficile à mettre en œuvre, car soumise à des jeux de pouvoir locaux et aux asymétries internes et externes entre les acteurs concernés. Elle n'en demeure pas moins importante pour rendre plus opérationnel le concept de développement durable, en Nouvelle-Calédonie, dans le Pacifique insulaire et ailleurs.

\section{BIBLIOGRAPHIE}

d'Aquino Patrick, Seck Sidi Mohamed., Bélières Jean-François, Passouant Michel, Sarr Malik, «Irrigation et développement régional : dix ans d'actions sur le fleuve Sénégal pour une planification régionale et décentralisée », Cahiers de la Recherche-Développement, vol. 45, 1999. p. 77-84.

d'Aquino Patrick, « SiSup : une expérience de suivi participatif des politiques de développement en Nouvelle Calédonie ", Communication au colloque CORAIL 2005. Territoire et Patrimoine en Océanie, Nouméa, Centre Culturel Jean-Marie Tjibaou, les 1, 2 et 3 décembre 2005.

d'Aquino Patrick, Le Page Christophe, Bousquet François, Bah Alassane,. « Using self-designed role-playing games and a multi-agent system to empower a local decision-making process for land use management: The SelfCormas experiment in Senegal ", Journal of Artificial Societies and Social Simulation, vol. $6 \mathrm{n}^{\circ}$ 3, 2003.

d'Aquino Patrick, « Gestion décentralisée de territoire : pour une animation inter-institutionnelle plutôt qu'un appui direct à la planification locale » dans L'Europe et le Sud à l'aube du 21e siècle : enjeux et renouvellement de la coopération, Actes de la $9^{\mathrm{e}}$ Conférence générale de l'Association Européenne des Instituts de Recherche et de Formation en matière de développement, Paris, EADI, 2002.

EchoGéo, 7 | 2008 
d'Aquino Patrick, Etienne, Michel Barreteau, Olivier, Le Page Christophe, Bousquet François, « Jeux de rôles et simulations multi-agents. » dans Modélisation des agroécosystèmes et aide à la décision, Montpellier, Cirad-INRA, collection Repères, 2001, p. 373-390.

Aubertin Catherine, Vivien Franck-Dominique, Le développement durable : enjeux politiques, économiques et sociaux, Paris, La Documentation française, coll. « Les études », 2006.

Bensa Alban, Bourdieu Pierre, «Quand les canaques prennent la parole », Actes de la Recherche en Sciences Sociales, vol. 56, 1985, p. 69-83.

Berr Eric, Harribey Jean-Marie, Le concept de développement en débat, Economie et sociétés, série $\mathrm{F}, \mathrm{n}^{\circ} 43,2005$.

Bouard Séverine, d'Aquino Patrick Sourisseau Jean-Michel, Pascutto Tamara. Enjeux sociaux, enjeux et développement : Expérimentations de méthodes participatives spécifiques pour favoriser les transformations sociales et l'adaptation des modèles de développement à la Nouvelle-Calédonie, Pouembout, IAC, 2006.

Bouard S., D’Aquino P., 2007. « Le jeu de rôles Ne Jèna : Une méthode participative pour discuter les pratiques de commercialisation des Mélanésiens. » Cahiers Agricultures. Vol. 16. n 6, p. 491-495.

Christnacht Alain, la Nouvelle-Calédonie, Les Etudes de la Documentation Française. 2004.

Collectif COMMOD. La modélisation comme outil d'accompagnement. Natures Sciences et Sociétés. 13, 2005, p. 65-168.

Commission Mondiale sur l'Environnement et le Développement (CMED), Notre avenir à tous, Editions du fleuve et Publications du Québec, 1987.

Davies Rick., Dart Jess, The Most Significant Change Technique. A Guide to its use, Care International, OXFAM, 2005. (www.mande.co.uk/docs/MSCGuide.htm).

Ehrlich Paul, "Key issues for attention from ecological economists", Environment and Development Economics, 13 p. 1-20, 2008.

Estrella Marisol, Blauert Jutta, Campilan Dindo, Gaventa John, Gonsalves Julian, Guijt Irene, Johnson Deb, Ricafort Roger, Learning from change. Issues and experiences in participatory monitoring and evaluation, Ottawa, IDRC, Participation in Development Series, 2000.

Figuière Catherine, Sud, développement et développement durable : l'apport de l'économie politique, Revue électronique de l'environnement Vertigo vol. 7, n² 2, septembre 2006.

Godard Olivier, Hubert Bernard, Le développement durable et la recherche scientifique à l'INRA. Rapport à Mme La Directrice Générale de l'INRA, 2002.

Godard Olivier, Le développement durable, une chimère malfaisante, La lettre de la prospective du Sénat, 08/10/2004.

Grossman Gene, Krueger Alan, « Economic Growth and the Environment », Quarterly Journal of Economics, 2, 1995, p. 353-377.

Harnmeijer Joanne, « From terms of reference to participatory learning: using an evaluation's creative space », PLA Notes, 36, 1999.

Harribey Jean-Marie. La lourdeur insoutenable du développement capitaliste, La Pensée, (331), juillet-septembre 2002.

Fetterman David, Kaftarian Shakeh, Wandersman Abraham, Empowerment evaluation knowledge and tools for self-assessment and accountability, SAGE, 1996. 
Latouche Serge, La Mégamachine. Raison technoscientifique, raison économique et mythe du progrès, La Découverte/MAUSS, 2004.

Rey-Valette Hélène, Roussel Sébastien, «L'évaluation des dimensions territoriale et institutionnelle du developpement durable : le cas des politiques de Gestion Intégrée des Zones Côtières ", Développement durable et territoire, Dossier 8 : Méthodologies et pratiques territoriales de l'évaluation en matière de développement durable, mis en ligne le 13 décembre 2006. URL : http://developpementdurable.revues.org/document3311.html.

Rist Gilbert, Le développement. Histoire d'une croyance occidentale. Paris, Presses de Sciences Po, Collection « Références inédites », 1996.

Rossi Gérard, L'ingérence écologique, Environnement et développement rural du Nord au Sud, CNRS, 2000.

Solow, Robert, “An Almost Practical Step toward Sustainability”, in Oates (ed.), The RFF Reader in Environmental and Resource Management, Washington DC, RFF, 1999, p. 263-272.

Sourisseau, Jean-Michel, Tyuienon, Raymond, Gambey, Jean-Claude, Djama, Marcel, Mercoiret, Marie-Rose, Sourisseau, Emmanuelle. Les Sociétés locales face aux défis du développement économique, Koné/Montpellier, IAC/Cirad/DDEE-PN. 2006.

Sourisseau, Jean Michel, Bouard, Séverine, « Stratégies des ménages ruraux et construction territoriale ; un éclairage par l'articulation des relations marchandes et non marchandes ", Communication au colloque CORAIL 2005. Territoire et Patrimoine en Océanie, Centre Culturel Jean-Marie Tjibaou, les 1, 2 et 3 décembre 2005.

Tubiana, Laurence, Environnement et développement. L'enjeu pour la France, Rapport au Premier Ministre, Paris, La Documentation française, 2000.

\section{NOTES}

1. L'archipel est le $3^{\mathrm{e}}$ producteur mondial de nickel, dont il détient les plus importantes réserves de la planète; deux projets de transformation de minerai, significatifs sur le marché mondiale sont par ailleurs sur le point d'aboutir, nécessitant l'intervention, nouvelle pour le pays, d'importantes firmes multinationales.

2. Une grande partie de sa barrière récifale est inscrite sur ces bases au patrimoine mondial de l'humanité.

3. Entre 1984 et 1988 , la Nouvelle-Calédonie a connu une situation de troubles graves et prolongés entre indépendantistes et loyalistes. La signature des accords de Matignon-Oudinot y mettra fin, en engagent les deux partis vers davantage d'autonomie dans le respect de toutes les communautés.

4. Dans le carré noir au dessus des personnages est inscrit l'effectif correspondant à chaque catégorie identifiée, à partir d'une enquête menée par l'équipe parallèlement à la réalisation participative du graphique (les effectifs affichés ne sont donc jamais définitifs et sont mis à jour au fur et à mesure de l'avancement de l'enquête). 


\section{RÉSUMÉS}

L'objet de cet article est d'explorer les conditions et l'opérationnalité d'une réflexion participative sur le développement durable et ses implications pour élaborer et évaluer des politiques publiques de développement. Après une présentation succincte des controverses dont fait l'objet le concept de développement durable à l'échelle mondiale, nous tenterons de préciser les enjeux de durabilité dans le contexte de la Nouvelle-Calédonie. L'entrée privilégie les articulations entre les trois dimensions de la durabilité, en s'appuyant sur les formes localement perçues de la performance économique, de l'intégrité de l'environnement et de l'équité sociale. Pour illustrer ces enjeux, nous nous intéressons plus précisément à la gestion sociale des impacts d'un important projet industriel d'extraction et de traitement du nickel dans la province Nord de Nouvelle-Calédonie. L'article décrit ensuite les principes à respecter pour la mise en place d'un suivi évaluation concerté entre les différents acteurs, susceptible de faciliter la prise en charge de cette transversalité et la gestion sociale des impacts du projet. Nous partons pour cela des acquis d'un programme de recherche portant sur l'élaboration d'une méthode de suivi évaluation participatif des programmes de développement local en province Nord. Enfin, nous insisterons sur les contraintes à l'efficacité et à la pérennité de dispositifs réellement participatifs, mais aussi à l'application des principes du développement durable, notamment dans le contexte du Pacifique insulaire.

The aim of this article is to investigate the conditions and the interests of a participatory reflexion on the sustainable development and its consequences to draw up and to assess development policies. After a brief display of the controversies carried out by the use and sense of the "sustainable development", we explain what is at stake about durability in the context of New Caledonia. We choose to focus on linkages between three dimensions of the durability. We start from the local perceptions of the economic performance, the integrity of the environment and the social equity. As an illustration of what is at stake in sustainable development policies, we present the social management of the impacts of an important industrial project of extraction and treatment of the nickel in the North province of New Caledonia. Considering that participatory follow-up evaluation facilitate transversality and the social management of the impacts of the project, the article describes the principles to respect for the implementation of a participatory follow-up evaluation. These principles are based on the experiences of a research program built around the evaluation of a grassroots development project in the northern province of New Caledonia. Finally, we insist on the constraints in the efficiency of real participatory device and on the difficulties to follow principles of the sustainable development, especially in the context of the Pacific islands.

\section{INDEX}

Mots-clés : démarches participatives, développement durable, Nouvelle-Calédonie, Politiques publiques

Keywords : New-caledonia, Participatory methods, Public policies, Sustainable development 


\section{AUTEURS}

\section{SÉVERINE BOUARD}

Séverine Bouard (severine.bouard@cirad.fr) est doctorante en Géographie à l'IAC-Cirad. Elle notamment publié

- Bouard, S., D’Aquino., P., 2007. « Le jeu de rôles Ne Jèna : Une méthode participative pour discuter les pratiques de commercialisation des Mélanésiens. » Cahiers Agricultures. Vol. 16. nº 6 . p. 491-495.

\section{JEAN-MICHEL SOURISSEAU}

Jean-Michel Sourissea (sourisseau@iac.nc) est chercheur en économie à l'IAC/Cirad-ES. Il a entre autres publié :

- Burnod P., Sourisseau JM., 2007. Changement institutionnel et immigration clandestine à Mayotte. Quelles conséquences sur les relations de travail dans le secteur agricole? Autrepart (43), 2007, p. 165-176.

- Sourisseau JM, Burnod P., Bonnal Ph., 2008. Changement institutionnel et agriculture à Mayotte. Les impacts d'une intégration renforcée à la République française sur l'activité des ménages. Economie Rurale 303-304-305, janvier-mai 2008. p 60-74.

\section{GILLES PESTANA}

Gilles Pestana (pestana@univ-nc.nc) est maître de conférences en géographie à l'université de la Nouvelle-Calédonie, UMR 8586 PRODIG. Parmi ses dernières publications figurent :

- Pestaña, G., 2008. Deux voisins dans la mondialisation : les relations économiques entre la Nouvelle-Zélande et la Nouvelle-Calédonie. in Levine S. and Angleviel F. ed., New Zealand - New Caledonia: Neighbours, Friends, Partners. Wellington, Victoria University Press, p. 280-301.

- Pestaña, G., 2006. L'État des géographes dans le Pacifique : la première figure de l'État c'est la carte! in De Deckker, P. dir. Les Figures de l'État dans le Pacifique. Paris, l'Harmattan \& Institut des Mondes Océaniens et Australasiens, p. 311-346. 